\title{
NIRCam coronagraphic Lyot stop: design, fabrication, and testing
}

\author{
Yalan Mao, Torben B. Andersen, Tracy Kubo, Miguel \\ Virgen, Henry Chan, et al.
}

Yalan Mao, Torben B. Andersen, Tracy Kubo, Miguel Virgen, Henry Chan, Greg Feller, Lynn W. Huff, Eric Smith, Gopal Vasudevan, Steve Somerstein, Tom Jamieson, Scott Horner, John Krist, Charles A. Beichman, Carmen Barone, Ron Schmidt, Donna Levin, Steve Seymour, Douglas Kelly, Marcia J. Rieke, "NIRCam coronagraphic Lyot stop: design, fabrication, and testing," Proc. SPIE 8150, Cryogenic Optical Systems and Instruments XIII, 81500E (28 September 2011); doi: 10.1117/12.896451

EDent: SPIE Optical Engineering + Applications, 2011, San Diego, California, United States 


\title{
NIRCam Coronagraphic Lyot Stop: Design, Fabrication and Testing
}

\author{
Yalan Mao ${ }^{\mathrm{a}}$, Torben B. Andersen ${ }^{\mathrm{a}}$, Tracy Kubo ${ }^{\mathrm{a}}$, Miguel Virgen ${ }^{\mathrm{a}}$, Henry Chan ${ }^{\mathrm{a}}$, Greg \\ Feller $^{\mathrm{a}}$, Lynn W. Huff ${ }^{\mathrm{a}}$, Eric Smith ${ }^{\mathrm{a}}$, Gopal Vasudevan ${ }^{\mathrm{a}}$, Steve Somerstein ${ }^{\mathrm{a}}$, Tom \\ Jamieson $^{\mathrm{a}}$, Scott Horner ${ }^{\mathrm{b}}$, John Krist ${ }^{\mathrm{c}}$, Charles A. Beichman ${ }^{\mathrm{c}}$, Carmen Barone ${ }^{\mathrm{d}}$, Ron \\ Schmidt $^{\mathrm{d}}$, Donna Levin ${ }^{\mathrm{d}}$, Steve Seymour ${ }^{\mathrm{d}}$, Douglas Kelly ${ }^{\mathrm{e}}$, Marcia J. Rieke ${ }^{\mathrm{e}}$ \\ a Advanced Technology Center, Lockheed Martin Space Systems Company, 3215 Porter Dr., Palo Alto, \\ CA 94304 \\ ${ }^{\mathrm{b}}$ NASA AMES, Sunnyvale, CA 94089 \\ c Jet Propulsion Laboratory/California Institute of Technology, 4800 Oak Grove Drive, Pasasdena, CA \\ 91109 \\ ${ }^{\mathrm{d}}$ Max Levy Autograph, Inc., 2710 Commerce Way, Philadelphia, PA 19154 \\ ${ }^{\mathrm{e}}$ Steward Observatory, University of Arizona, Tucson, AZ 85721
}

\begin{abstract}
The NIRCam instrument on the James Webb Space Telescope (JWST) will provide a coronagraphic imaging capability to search for extrasolar planets in the $2-5$ microns wavelength range. This capability is realized by a set of Lyot pupil stops with patterns matching the occulting mask located in the JWST intermediate focal plane in the NIRCam optical system. The complex patterns with transparent apertures are made by photolithographic process using a metal coating in the opaque region. The optical density needs to be high for the opaque region, and transmission needs to be high at the aperture. In addition, the Lyot stop needs to operate under cryogenic conditions. We will report on the Lyot stop design, fabrication and testing in this paper.
\end{abstract}

Keywords: JWST, NIRCam, Coronagraphic Imaging, Lyot Stop

\section{INTRODUCTION}

The JWST will provide a capability to search for extrasolar planets in the wavelength range $2-5$ microns using the NIRCam ${ }^{1}$ instrument on the JWST. NIRCam has a coronagraphic imaging mode, which is realized by employing a combination of occulting masks located in the intermediate image plane, with a Lyot stop located at the NIRCam pupil plane. The occulting masks, sized for specific wavelengths chosen to optimize planet detection, will block the central portion of a star's point spread function (PSF) at the JWST telescope focus with different sized spot and wedge-shaped patterns (see Figure 1). The detailed occulting mask design and fabrication have been described by Krist et al. ${ }^{2}$ The Lyot stops complete the suppression of the stellar PSF by masking any starlight that bypasses the occulters.

In this paper, we will report on the Lyot stop design, manufacturing and testing.
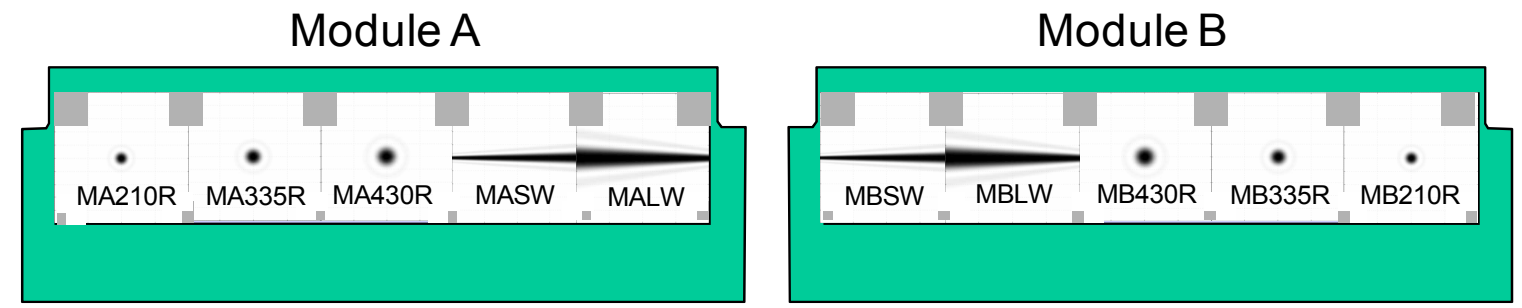

Figure 1. Occulting masks in the two NIRCam Modules

Cryogenic Optical Systems and Instruments XIII, edited by James B. Heaney, E. Todd Kvamme Proc. of SPIE Vol. 8150, 81500E · C 2011 SPIE · CCC code: 0277-786X/11/\$18 · doi: 10.1117/12.896451 


\section{LYOT STOP DESIGN}

The diffraction pattern in the NIRCam pupil formed by a star centered on an occulting mask consists of the remaining starlight concentrated around the images of the telescope obscurations (segment edges, secondary obscuration and spiders) and contains areas (holes) of very low intensity. By placing an opaque mask in the pupil with holes corresponding to these low-intensity areas, almost all light from the occulted star will be suppressed, while that from an offset planet slips through the holes. Rather than placing the mask at the telescope exit pupil, it will be located in the filter wheel where the pupil is relayed by the NIRCam collimator. Figure 2 shows the predicted pattern in the pupil formed by a star behind the spot occulter (circular shape in Figure 1) and wedge occulter (arrow shape in Figure 1). The corresponding Lyot stops are depicted in Figure 2 (c) for the spot occulter and Figure 2 (d) for the wedge occulter.

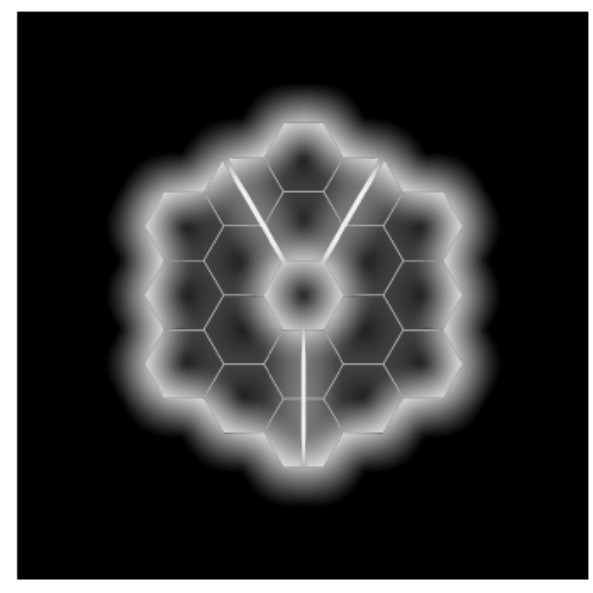

(a) Spot occulter diffraction

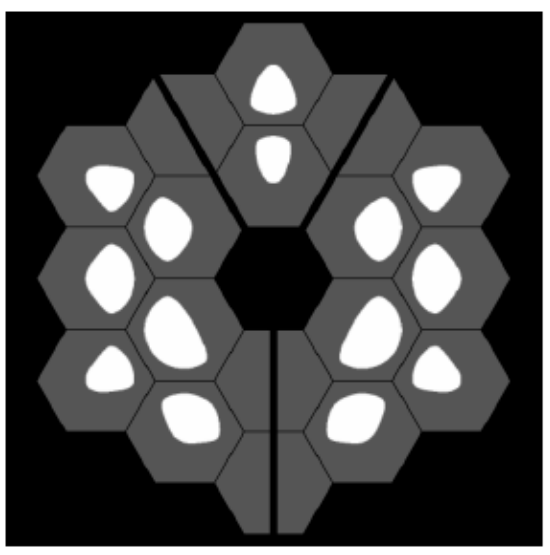

(c) Spot occulter Lyot stop

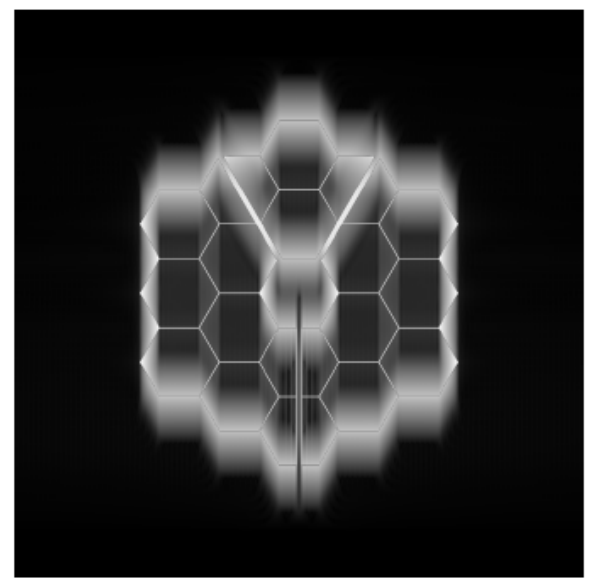

(b) Wedge occulter diffraction

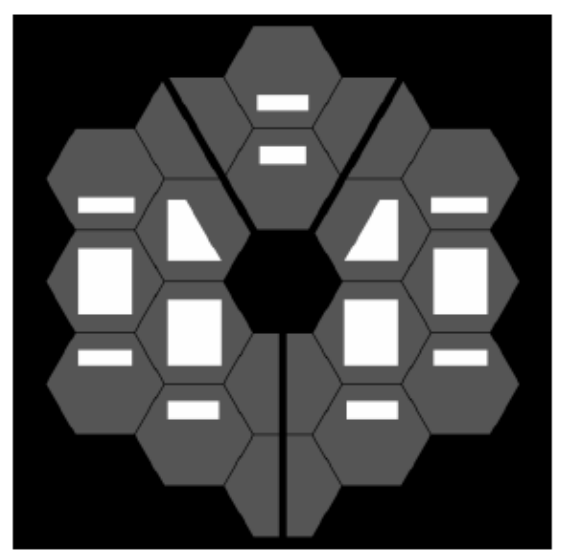

(d) Wedge occulter Lyot stop

Figure 2. (a) diffraction pattern in the telescope exit pupil formed by a spot occulter; (b) diffraction pattern in the telescope exit pupil formed by a wedge; (c) ideal Lyot stop for the spot occulter; (d) ideal Lyot stop for the wedge occulter, shown as white apertures superposed on the JWST pupil (grey and black). 


\subsection{Optical modeling}

The locations of the holes in the Lyot stops in an ideal system were derived by computing the diffraction pattern at the pupil for an occulted source using the PROPER wavefront propagation library ${ }^{3}$. Because the actual NIRCam pupil image is distorted due to design constraints, the coordinates of the holes had to be converted to match. This was accomplished by ray tracing with Lockheed Martin's in-house software package Optima ${ }^{4,5}$ using a model for the combined NIRCam shortwave (SW) and longwave (LW) channels. In the optical model, the Lyot stop surface is identified as the first surface of pupil in each channel SWP 1 or LWP 1. The global coordinates to the center of the Lyot stop surface in both optical model coordinates $(\mathrm{X}, \mathrm{Y}, \mathrm{Z})$ and in space craft coordinates $(\mathrm{V} 1, \mathrm{~V} 2, \mathrm{~V} 3)$ are identified.

The sapphire plate substrate at the coronagraph occulting mask (COM) location was modeled as cold sapphire, using the ordinary refractive index for sapphire at $37 \mathrm{~K}$ calculated using the Tropf model of Yang et al. ${ }^{6}$ The extraordinary rays were also traced. No significant differences were found between the o-ray and e-ray trace.

To establish the Filter Wheel assembly coordinate system F[F1, F2, F3], a dummy surface was inserted in contact with the SWP 1 (LWP 1) surface; this surface was then rotated around its surface normal through the local vertex until its local F2-axis was exactly perpendicular to the global YZ-plane and so that the local F3-axis pointed in the direction of light propagation at the surface.

There are two Lyot stops in each optical path (channel). One Lyot stop is the spot stop, the other one is the wedge stop. There are two spot occulters (4.3 and 3.35 microns) in the LW channel. Only one can be used in one channel. The arrangements are listed in Table 1 . The wavelength selection is physically realized by the bandpass filters located in the NIRCam Filter Wheel. The spectral band of the each bandpass filter are given by Mao et $\mathrm{al}^{7}$

For each path (SW or LW) and each mask type (Wedge or Spot), the relevant field point and wavelength were chosen and rays were then traced in Optima aimed at the scaled outline of each Lyot stop hole on the JWST telescope exit pupil and the $(\mathrm{F} 1, \mathrm{~F} 2)$ ray intersections at the Lyot stop surface (the surface labeled SWP 1 or LWP 1, respectively) were recorded. For the 4-sided wedge holes, the outline was created by generating 50 points between neighboring polygon vertices and tracing rays to all these points. For the curved holes in the spot occulter's Lyot stop, more than 200 points were used for each stop hole. The coordinates of the Lyot stops are plotted in Figures 3 and 4 for the spot stop and wedge stop, respectively. The spot stops of SW Module A and Module B in Figure 3 are mirror images of each other. All the other Lyot stops are unique.

Table 1. Lyot stop wavelength selection for each optical path

\begin{tabular}{cc}
\multicolumn{3}{c}{ Mask centers, } & Module A \\
XAN(deg) & YAN(deg) \\
0.01320 & -0.01839 \\
0.01875 & -0.01839 \\
0.02431 & -0.01839 \\
0.02987 & -0.01839 \\
0.03542 & -0.01839 \\
& \\
Mask centers, & Module B \\
XAN(deg) & YAN(deg) \\
-0.01320 & -0.01839 \\
-0.01875 & -0.01839 \\
-0.02431 & -0.01839 \\
-0.02987 & -0.01839 \\
-0.03542 & -0.01839
\end{tabular}

$\begin{array}{rrr}\text { Lyot Stop Design, Module A } \\ \text { XAN(deg) } & \text { YAN(deg) Wavelength (micron) } \\ 0.01320 & -0.01839 & 4.6 \\ 0.01875 & -0.01839 & 2.1 \\ 0.02431 & -0.01839 & 4.3 \\ & & \mathrm{~N} / \mathrm{A} \\ 0.03542 & -0.01839 & 2.1 \\ & & \\ \text { Lyot Stop Design, Module B } & \\ \text { XAN(deg) } & \text { YAN(deg) Wavelength (micron) } \\ -0.01320 & -0.01839 & 2.1 \\ -0.01875 & -0.01839 & 4.6 \\ & & \mathrm{~N} / \mathrm{A} \\ -0.02987 & -0.01839 & 3.35 \\ -0.03542 & -0.01839 & 2.1\end{array}$


To verify the analysis in Optima, we also performed optical analysis using $\mathrm{CodeV}^{8}$ for one of the masks. The results for this one mask from Optima and CodeV agree very well.

\subsection{Lyot stop optic wedge design}

The field points corresponding to the locations of the occulting masks are outside the NIRCam focal plane array (FPA) for normal imaging. Figure 5 shows the NIRCam in-field images inside the FPA and the Coronagraphic images outside the FPA. In order to bring the out-side field of view into the FPA, a wedge design is incorporated in the Lyot stop optic. Figure 6 shows that the Barium Fluoride $\left(\mathrm{BaF}_{2}\right)$ wedge $\left(5^{\circ}\right.$ wedge) design with curvature on one surface is best among several design trades for the SW path. The dispersion is at a minimum with the $\mathrm{BaF} 2$ wedge design when three wavelengths (shortest, middle and longest in each filter band) are modeled for each filter band. A Silicon wedge $\left(1^{\circ}\right)$ was selected for the LW path. The optic wedge orientation relative to Lyot stop hole patterns has been clearly marked in Figure 3 and 4. Figure 7 shows coronagraph wedge pupil wheel orientations for Module A, Module B, SW \& LW.

\subsection{Intensity suppression and optical density}

We have set our design goal of suppression at the Lyot stop to be better than $10^{-6}$ (OD6). Figure 8 shows some candidate metal coatings on the $\mathrm{BaF}_{2}$ substrate. Similar behavior has been found for the Silicon substrate.

\subsection{Tolerance considerations}

At $\lambda=4.6 \mu \mathrm{m}$, the contrast degrades by $0.1 \mathrm{x}-0.2 \mathrm{x}$ for a $2 \%$ pupil shear, but it degrades by $\sim 10 \mathrm{x}$ for a $3 \%$ pupil shear. Therefore, the Lyot stop hole vertices need to be accurate to $\pm 0.03 \mathrm{~mm}$ to accommodate a pupil shear of $2 \%$ of the pupil diameter.

\section{LYOT STOP FABRICATION}

\subsection{Lyot Stop Wedge Substrates}

The chosen Lyot stop wedge substrates are $\mathrm{BaF}_{2}$ for $\mathrm{SW}$ and $\mathrm{Si}$ for $\mathrm{LW}$ as stated in section 2.2. The shape of the Lyot stop substrates is depicted in Figure 7 with three flat cuts for mounting in the NIRCam Pupil Wheel. A circular shape may slip, cause rotational error and introduce Lyot stop pupil shear to the diffraction pattern generated by a star and nearby planet. As stated in section 2.4, a 3\% pupil shear will cause the contrast to degrade by $\sim 10 \%$. In order to prevent rotational slip in the Pupil Wheel due to pupil shear tolerance considerations, the Lyot stop wedge substrates were cut flat at three sides.

\subsection{Patterning Lyot Stop by Photolithography}

The Lyot stop hole patterning is fabricated by a photolithographic technique. The photolithographic technique will produce the Lyot stop hole vertices with an accuracy less than $\pm 0.015 \mathrm{~mm}$, which is half of the required tolerance indicated in section 2.2. Before depositing the masks on the flight optical wedges, the photomasks were first fabricated and tested with $\mathrm{BaF}_{2}$ coupons. The patterning process was carefully designed and developed to withstand repeated cryo-cycling. The early development coupons were cryo cycled to ensure survivability and retention of registration precision under cryogenic temperatures, by tuning in and perfecting the photolithography process. Several process iterations combining AR coating and metal coating refinement were performed. $\mathrm{BaF}_{2}$ is a tricky material to work with. The $\mathrm{BaF}_{2}$ substrate is a single crystal material, very sensitive to temperature, and can fracture easily with slight strains caused by temperature gradients and mechanical stresses. 


\subsection{Coatings on the Lyot stop}

AR coatings were applied on both sides of the Lyot stop wedge substrate. The AR coating has been found to be both an anti-reflective and adhesive material in the photolithography process. Without the proper AR coating, the metallic pattern produced by photolithography would not properly adhere to the Lyot stop wedge after cryogenic to ambient temperature cycling.

Several metal coating samples were produced and tested under cryogenic conditions. The tape (3M Scotch brand \#600 clear tape) pull tests were applied to the samples for the adhesion test. Some material combinations failed the adhesion tape test after the cryo-cycling. Some samples showed discoloration after the cryo-cycling. A gold coating with proper binding layer was selected for the Lyot stop for the high optical density coating.

Module A
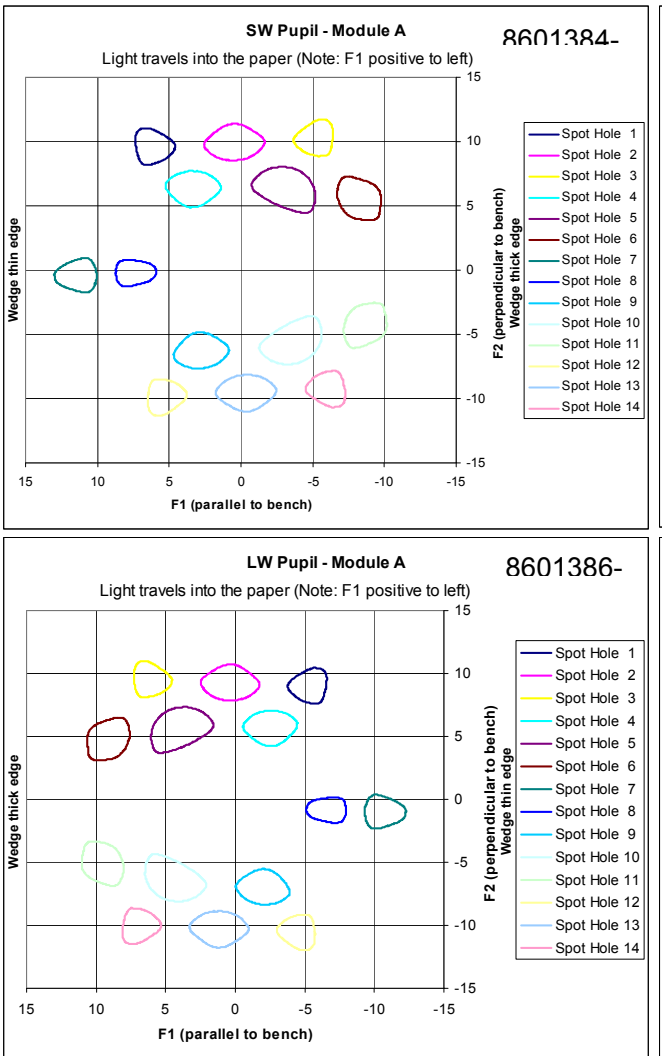

Figure 3. Coordinates of spot stops of the Lyot stop. From left to right: SW (upper)/LW (lower) Mod A, SW (upper)/LW (lower) Mod B.

Module B

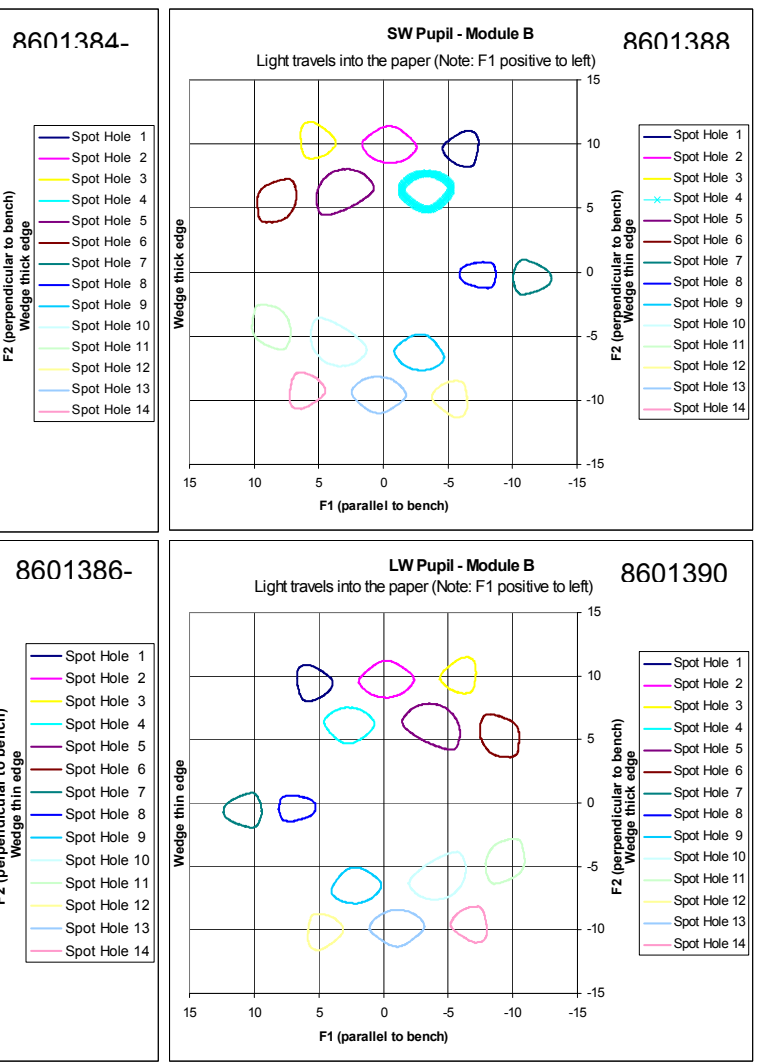


Module A
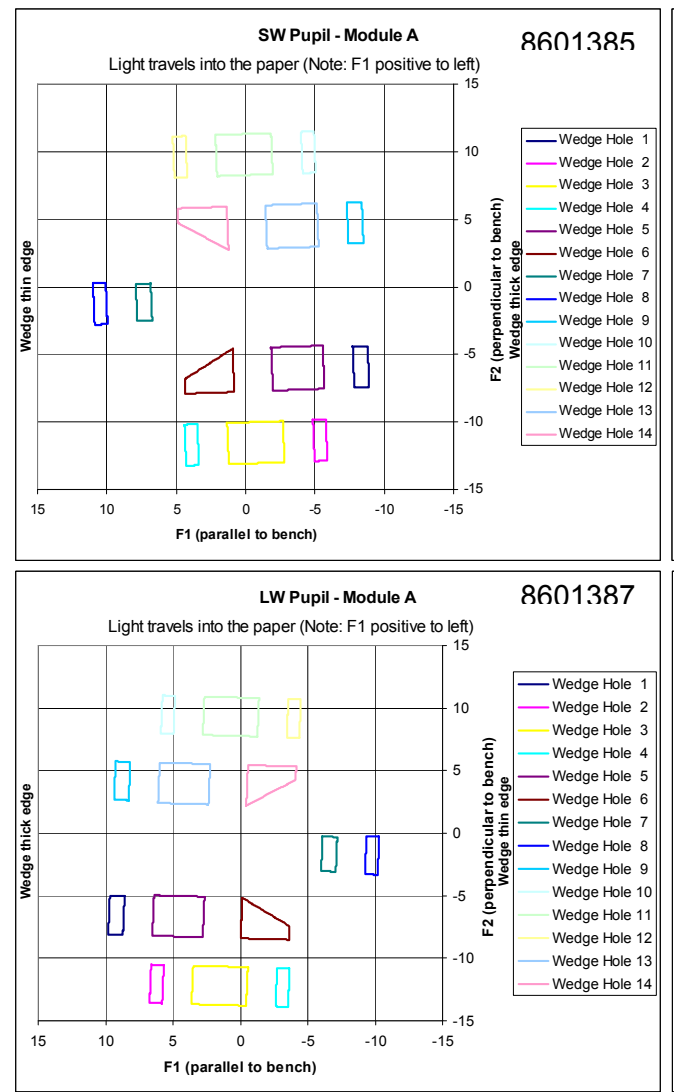

Module B
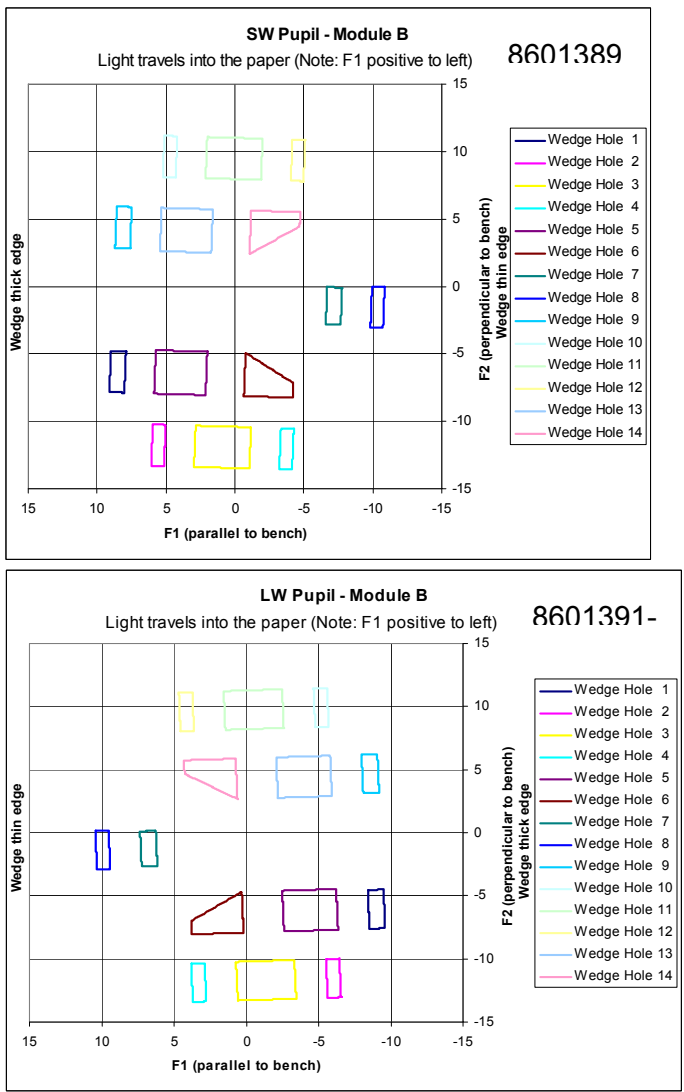

Figure 4. Coordinates of wedge spot stops of the Lyot stop. From left to right: SW (upper)/LW (lower) Mod A, SW (upper)/LW (lower) Mod B.

\section{LYOT STOP TESTING}

\subsection{Lyot Stop Cryogenic Environmental Testing}

Two completed Lyot stops (see Figure 9) went through two cryo-cycles from ambient to 30K. The tape test was applied to them after the two cryo-cycles. Both parts survived the cryo-cycling and the subsequent tape test. The temperature profile of the cryo-cycle is plotted in Figure 9. The witness samples had temperature sensors attached to them to record the temperature during cool-down and warm-up. 


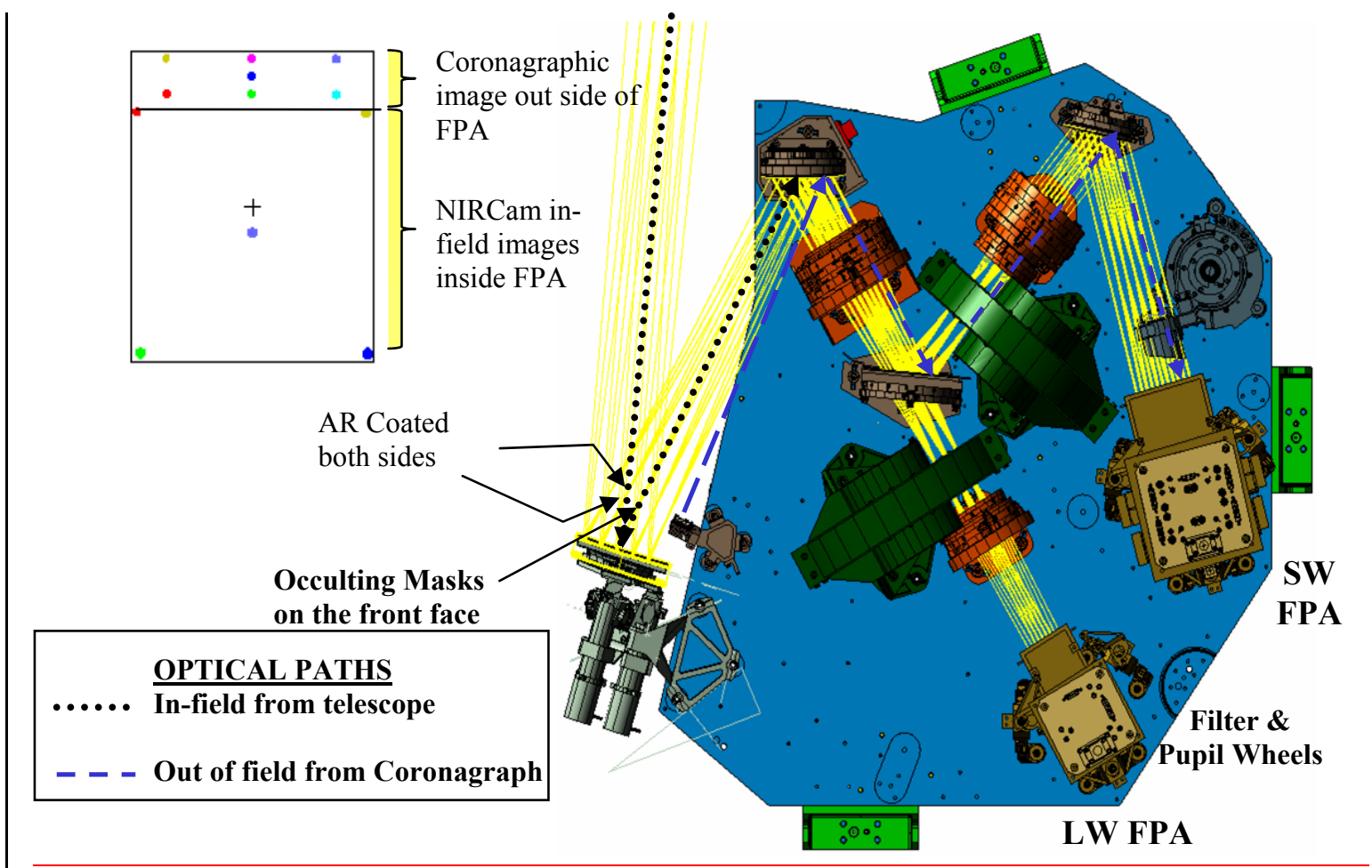

Figure 5. Left: NIRCam in-field image and coronagraph out-field image; Right: NIRCam optical layout
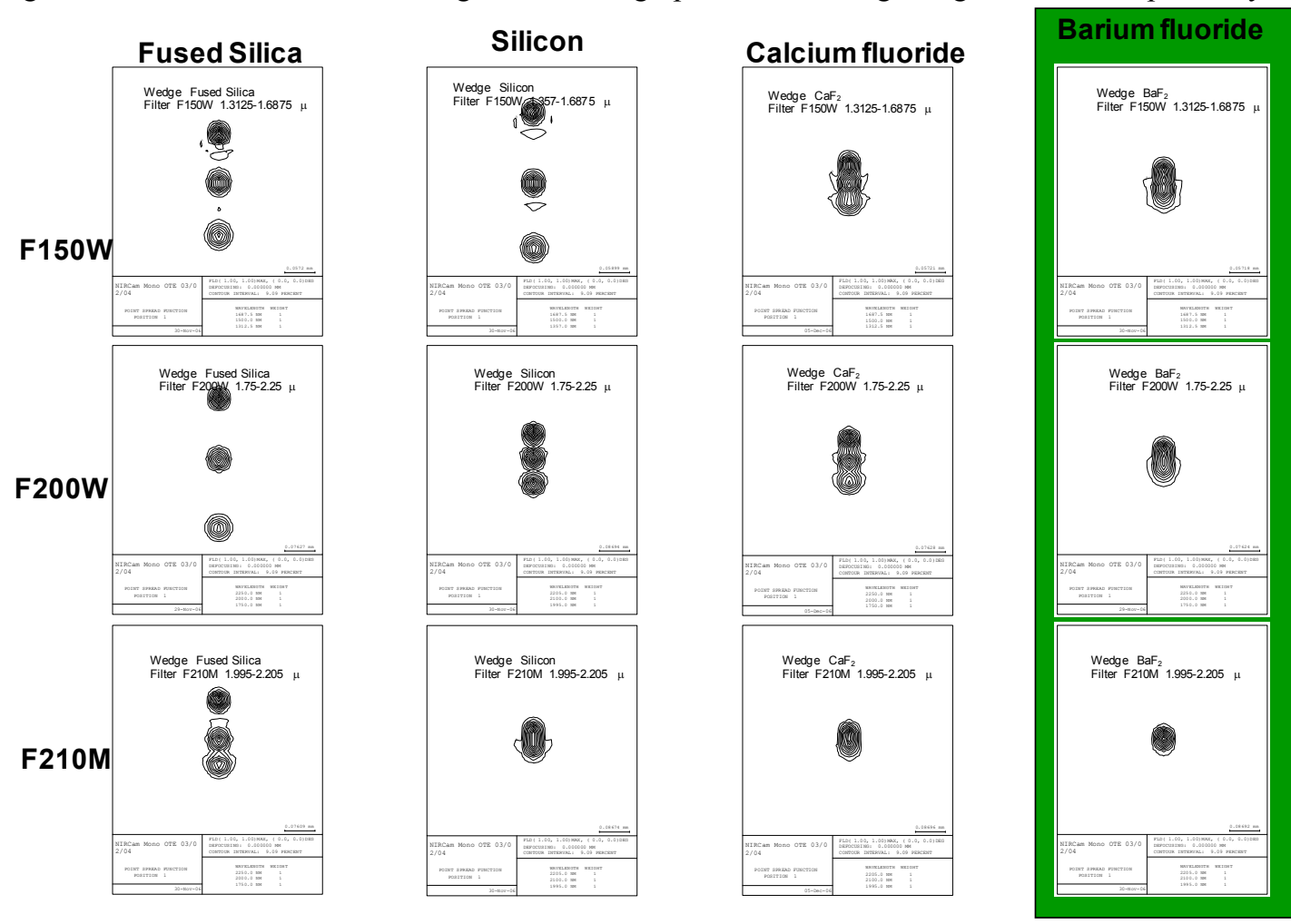

Figure 6. Design trades of Wedge-Induced Dispersion on the SW FPA 


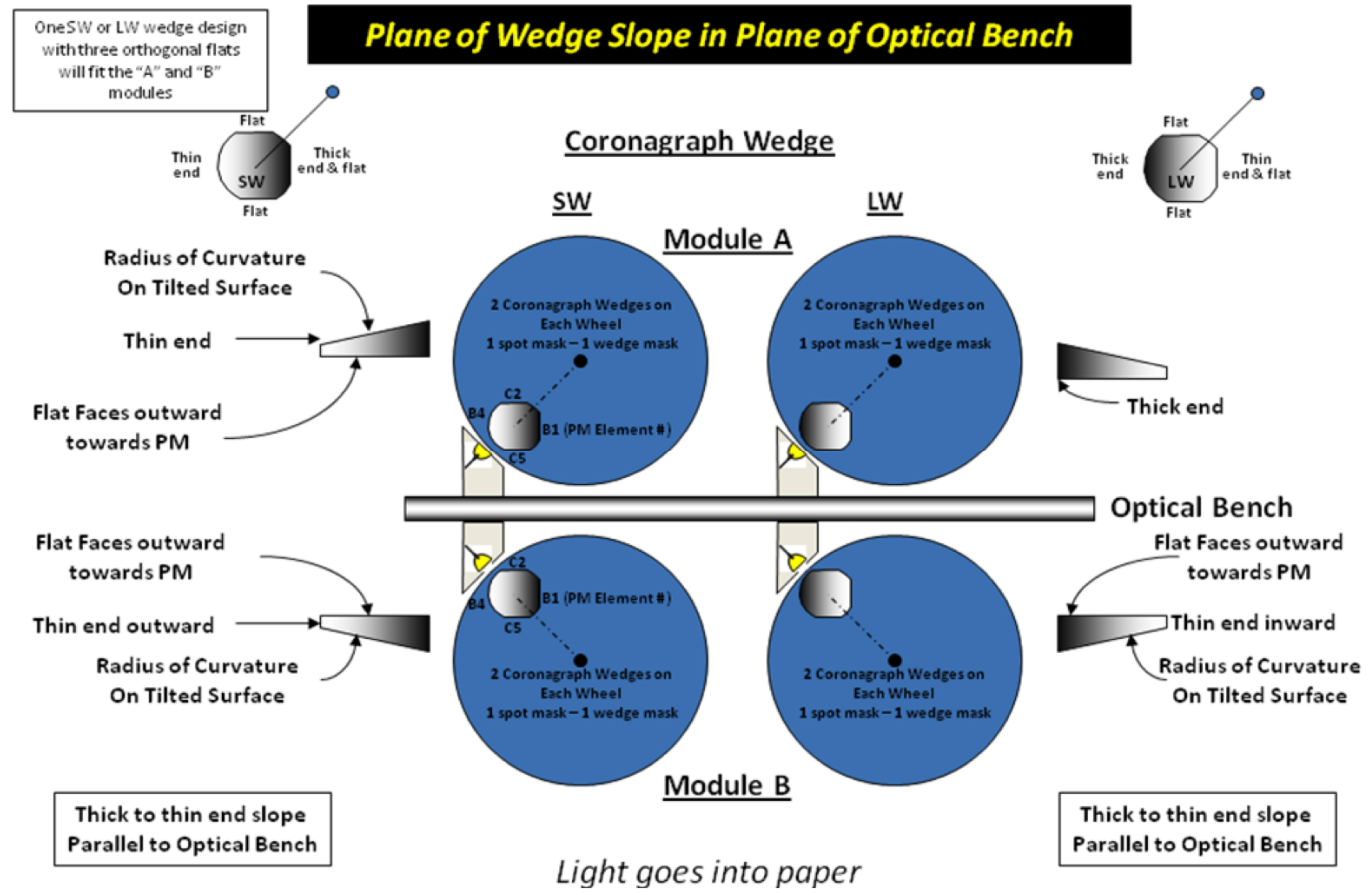

Figure 7. Coronagraph Wedge, Pupil Wheel Orientations, Modules A \& B, SW \& LW

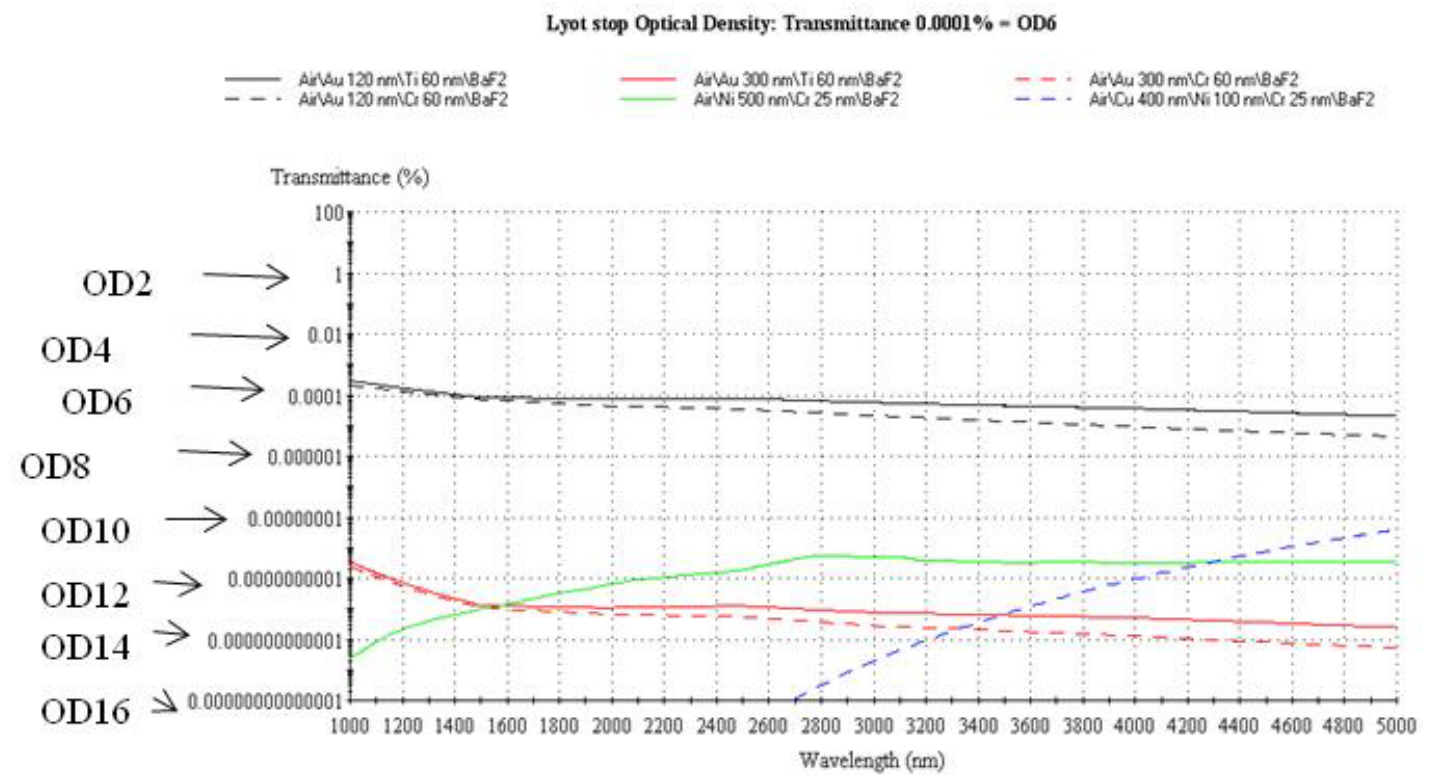

Figure 8. Blocking designs for Lyot stop with optical density better than OD6 for 2.0 - 5.0 microns. 

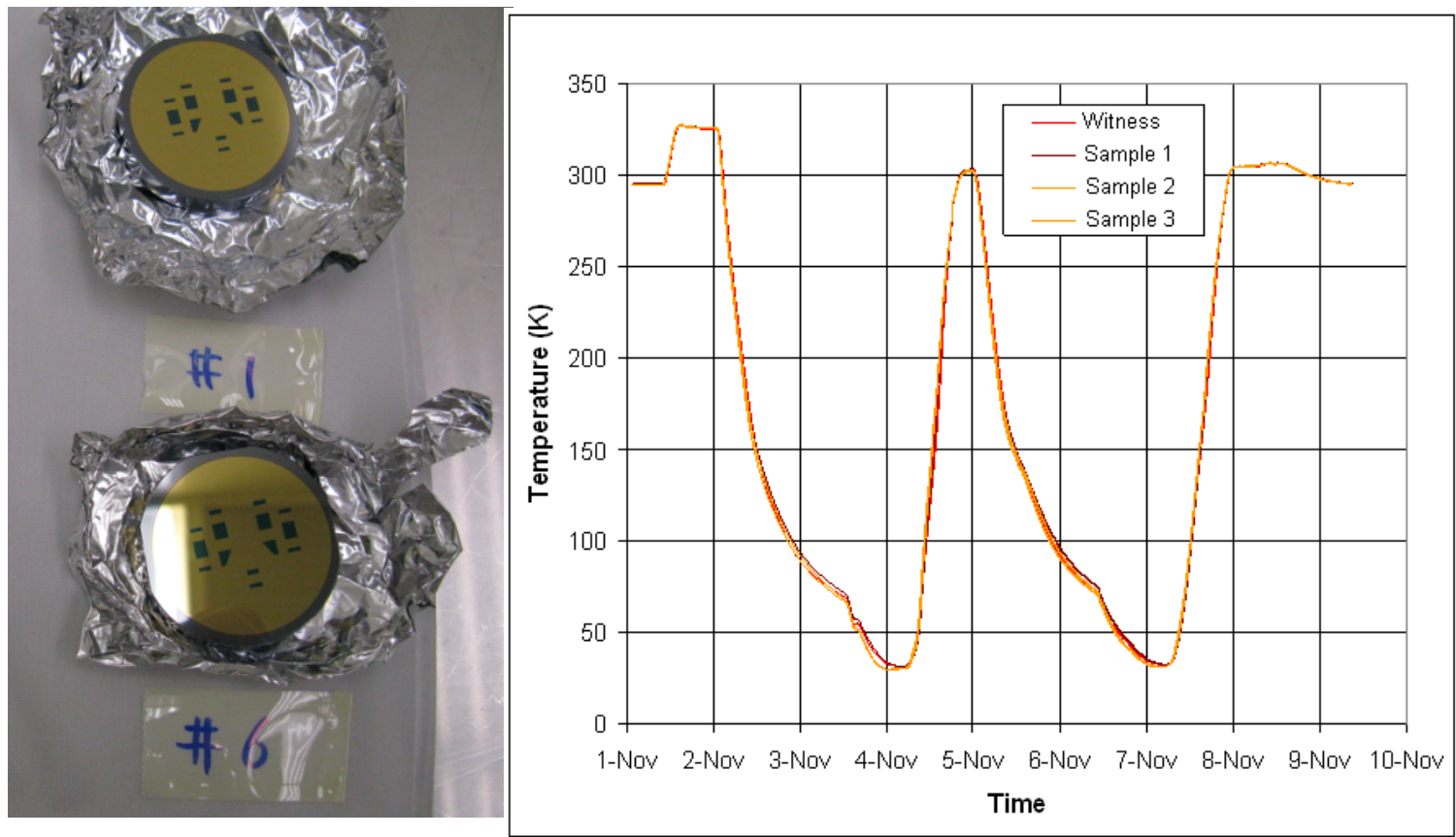

Figure 9. Two Lyot stops and the cryo-cycle temperature profile

\subsection{Lyot Stop Hole Vertices Measurement}

Lyot stop hole vertex measurements were performed on the photomasks. The positions of the Lyot stop hole vertex locations on the phototooling were measured with respect to the $\mathrm{X}$ and $\mathrm{Y}$ center lines of the wedges. The measurements recorded had an accuracy of $\pm 0.010 \mathrm{~mm}$. The OPTEK Inspection System used to measure each location is traceable to MLA Glass Scale NIST Test Number 821/262115-99. The procedures used are in compliance with MIL-STD-45662A. The methodology was to take four evenly spaced points around each stop location and confirm their positions. Table 2 lists the measurements of two holes on one of the spot stop masks and one of the wedge stop masks. The delta $\mathrm{X}$ and delta $\mathrm{Y}$ are the differences between the measurement and design vertex values. The differences are in the range \pm 0.011 $\mathrm{mm}$. The fabrication differences from the photomasks to the real parts were within $\pm 0.001 \mathrm{~mm}$. Therefore, the Lyot stop hole vertices were fabricated well within the required $\pm 0.015 \mathrm{~mm}$ requirement.

Table 2. Lyot Stop Hole Vertex Photomask Measurements

\begin{tabular}{|c|c|c|c|c|c|c|c|c|c|c|c|c|c|}
\hline \multicolumn{7}{|c|}{ SPOT STOP MASK: Clear Opening Area \# 1} & \multicolumn{7}{|c|}{ WEDGE STOP MASK: Clear Opening Area \# 1} \\
\hline pt\# & F1(SW) & F2(SW) & Measured & Measured & Delta X & Delta Y & pt\# & F1(SW) & F2(SW) & ured & Meas & Delta X & Delta Y \\
\hline 1 & 7.298 & 9.514 & 7.303 & 9.517 & 0.005 & 0.003 & 1 & -7.725 & -4.421 & -7.727 & -4.416 & -0.002 & 0.005 \\
\hline 53 & 6.193 & 8.235 & 6.186 & 8.236 & -0.007 & 0.001 & 51 & -8.808 & -4.381 & -8.799 & -4.378 & 0.009 & 0.003 \\
\hline 106 & 4.598 & 9.699 & 4.609 & 9.703 & 1 & 0. & 101 & -8.930 & -7.427 & 24 & -7 & 6 & 0.004 \\
\hline 159 & 6.458 & 11.048 & 6.462 & 11.045 & 0.004 & -0.003 & 151 & -7.848 & -7.466 & -7.846 & -7.467 & 0.002 & -0.001 \\
\hline \multicolumn{7}{|c|}{ SPOT STOP MASK: Clear Opening Area \# 2} & \multicolumn{7}{|c|}{ WEDGE STOP MASK: Clear Opening Area \# 2} \\
\hline t\# & $\begin{array}{c}\text { F1(SW) } \\
(\mathrm{mm})\end{array}$ & $\begin{array}{l}\text { F2(SW) } \\
(\mathrm{mm})\end{array}$ & $\begin{array}{c}\text { Measured } \\
X(\mathrm{~mm})\end{array}$ & $\begin{array}{c}\text { Measured } \\
\mathrm{Y}(\mathrm{mm})\end{array}$ & $\begin{array}{c}\text { Delta X } \\
(\mathrm{mm})\end{array}$ & $\begin{array}{c}\text { Delta } Y \\
(\mathrm{~mm})\end{array}$ & pt\# & $\begin{array}{l}\text { F1(SW) } \\
(\mathrm{mm})\end{array}$ & $\begin{array}{l}\text { F2(SW) } \\
(\mathrm{mm})\end{array}$ & $\begin{array}{c}\text { Measured } \\
X(\mathrm{~mm})\end{array}$ & $\begin{array}{l}\text { Measured } Y \\
(\mathrm{~mm})\end{array}$ & $\begin{array}{c}\text { Delta X } \\
(\mathrm{mm})\end{array}$ & $\begin{array}{c}\text { Delta } Y \\
(\mathrm{~mm})\end{array}$ \\
\hline 1 & 2.569 & 9.772 & 2.575 & 9.774 & 0.006 & 0.002 & 1 & -4.848 & -9.862 & -4.858 & -9.863 & -0.010 & -0.001 \\
\hline 62 & 0.520 & 8.506 & 0.510 & 8.504 & -0.010 & -0 . & 51 & -5.776 & -9.828 & -5 & -9.8 & 7 & 0.00 \\
\hline 125 & -1.648 & 9.852 & -1.642 & 9.848 & 06 & -0 & 101 & -5.899 & -12.869 & 99 & -12.868 & 0.000 & 0.00 \\
\hline 187 & 0.414 & 11.415 & 0.404 & 11.417 & -0.010 & 0.002 & 151 & -4.972 & -12.903 & -4.968 & -12.900 & 0.004 & 0.003 \\
\hline
\end{tabular}

\subsection{Lyot Stop Spectral Transmission Measurement}


The Lyot stop spectral performance was measured in hole area transmission and non-hole area transmission separately. The Lyot stop wedge substrates were first AR coated both sides. The Lyot stop hole spectral transmissions were measured after both sides were AR coated before the patterning process. Figure 10 (a) shows the spectral transmission of the $\mathrm{SW}$ coating on the $\mathrm{BaF}_{2}$ substrate. The transmission is shown to be $\sim 80 \%$ in the wavelength 2.1 micron range. Figure (b) shows the spectral transmission of the LW multilayer AR coating on the Silicon substrate. The transmission is shown to be $98.1 \%$ at wavelengths 3.35 and 4.3 microns, and $98.2 \%$ at 4.6 microns.

The spectral transmission of the Lyot stop non-hole area is shown in Figures 10 (c) and (d).
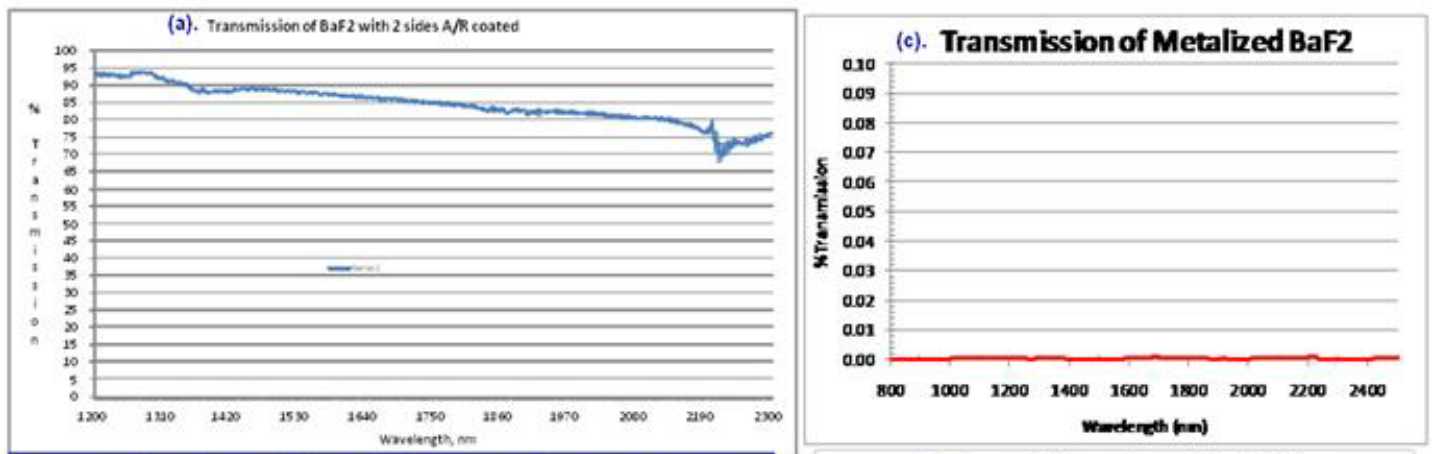

(b). Transmission of Silicon with 2 sides multi-layer AR coating
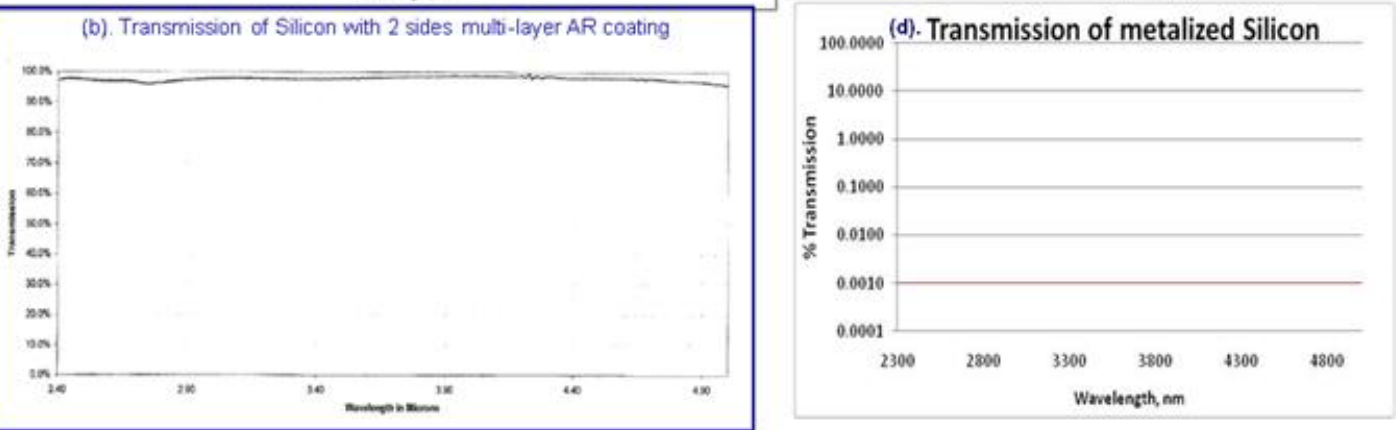

Figure 10. Lyot stop spectral transmission measurement results

The transmission is shown to be less than $0.025 \%$ from 880 to $2500 \mathrm{~nm}$ for the $\mathrm{SW}$, and from 2300 to $5000 \mathrm{~nm}$ for the $\mathrm{LW}$ channel. The measurement instrument is not capable of measuring the transmission to $0.0001 \%$, which corresponds to OD6. However, the transmission spectra shown in Figures 10 (c) and (d) indicate a better than OD4 flat level in the spectra in the wavelength range measured.

\subsection{Lyot Stop Optical Density Measurement}

The Lyot stop optical density (OD) is an important parameter. The spectral transmission measurement instrument is not capable of measuring to the OD6 level, as discussed above. However, the measurements showed the spectral flatness of better than OD4 for both the SW and LW channel wavelength ranges (see Figures 10 (c) and (d)). Based both on the spectral measurements and the design (see Figure 8), if we can measure at one wavelength with a laser and show the OD level to be better than OD6, we can conclude that the Lyot stop spectral suppression is better than OD6.

Figure 11 depicts the Lyot stop optical measurement setup. The laser was a Newport 744 Laser Module with Newport 505 Laser Driver and 325 Temperature Controller. The wavelength of the laser diode was $1550 \mathrm{~nm}$, collimated to a beam diameter of approximately $1 \mathrm{~mm}$. The Newport 918D-IR Detector was 
used with an OD3 ND Filter and Newport Power Meter 1918-C. The setup was first checked with various calibrated ND filters for the optical density measurement range, and periodically checked every few Lyot stops measurements. There were five measurement locations for each Lyot stop being measured, one in the center, and four (two opposing orthogonal pairs) on the outer side of the hole area, as depicted in Figure 11.

The measured optical densities for each type of Lyot stop were tabulated and listed in the table in Figure 11. The Input Power was measured to be $87.9 \mathrm{~mW}$ for most measurements, with an empty beam path. The Transmitted Power was measured to be in the range of a few $\mathrm{nW}$ in a dark room with the Lyot stop in the beam path at the location. The optical density at each location was calculated with the formula:

\section{Optical Density $=-\log _{10}$ (Transmitted Power / Input Power).}

The optical Densities of the Lyot stops were measured to be better than OD6 at five locations for all the Lyot stop types (see Table in Figure 11). The image of the SW spot stop in the Figure 11 is taken from the AR coating side.

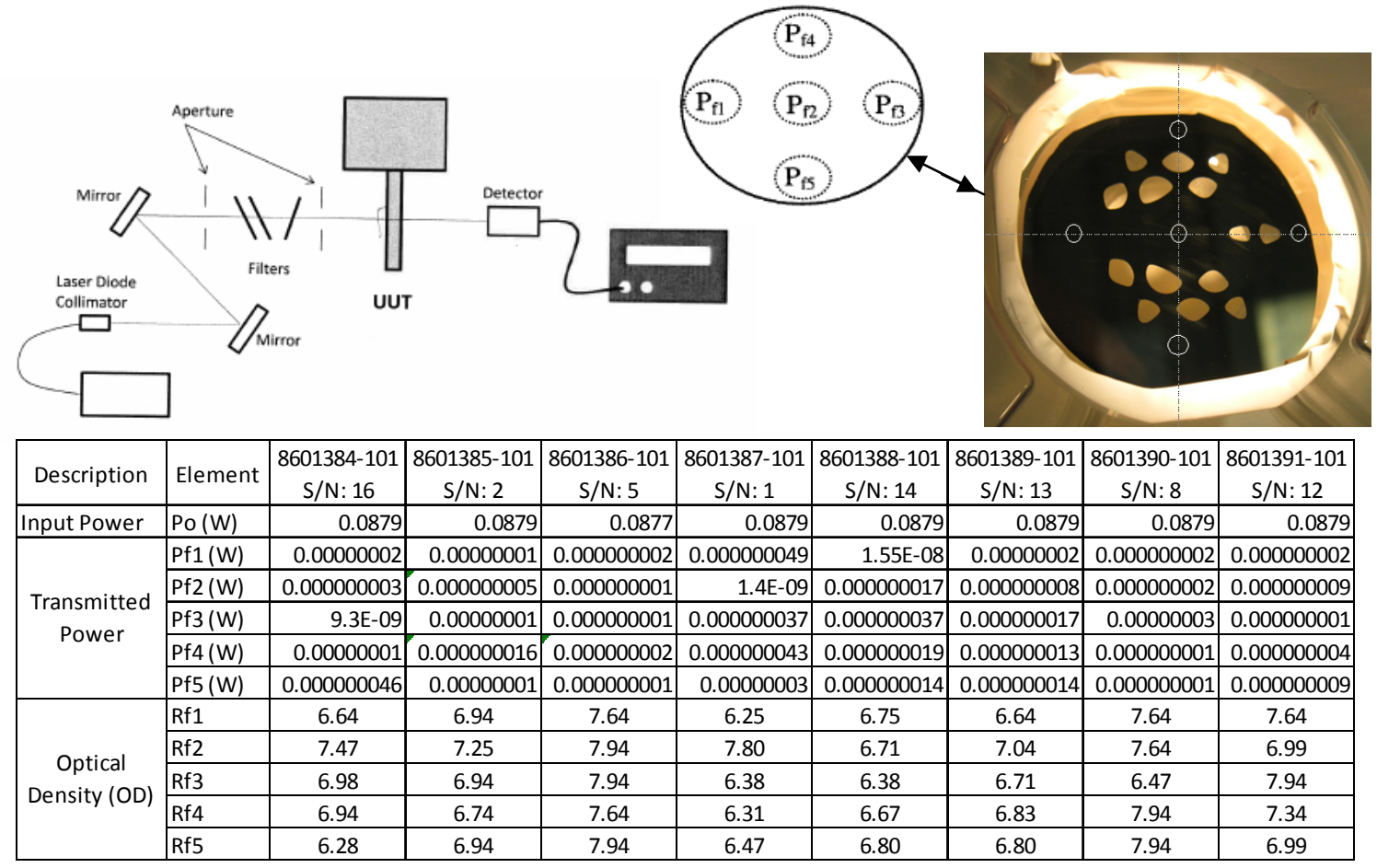

Figure 11. Lyot stop optical density measurement.

\section{CONCLUSION}

The Lyot stops in the NIRCam instrument have been designed, fabricated and tested to work at cryogenic temperatures. Eight different types of Lyot stops have been tested to have OD6 or denser at five locations on the Lyot stop opaque regions. The pupil shear of the Lyot stop hole vertices was tested to have tolerances well within $\pm 0.015 \mathrm{~mm}$, half of the $\pm 0.030 \mathrm{~mm}$ tolerance for a $2 \%$ pupil shear. All of those requirements combined to provide excellent coronagraphy for the NIRCam instrument. 


\section{ACKNOWLEDGEMENTS}

Development of the NIRCam instrument at the Lockheed Martin Advanced Technology Center is performed under contract to and teamed with the University of Arizona's Steward Observatory. The University of Arizona in turn is under contract to the JWST Project at the NASA Goddard Space Flight Center. This work was performed and funded by NASA Goddard Space Flight Center under Prime Contract NAS5-02105. Portions of this work were performed at the Jet Propulsion Laboratory/California Institute of Technology.

Many thanks to Jeff Vanden Beukel ${ }^{\mathrm{a}}$, Alison Nordt ${ }^{\mathrm{a}}$, Bruce Steakley ${ }^{\mathrm{a}}$, Richard Vassar ${ }^{\mathrm{a}}$, Michael Jacoby ${ }^{\mathrm{a}}$, Todd Kvamme ${ }^{\mathrm{a}}$, Bela Privari ${ }^{\mathrm{a}}$, Charles Clark ${ }^{\mathrm{a}}$, Yelena Guralnik ${ }^{\mathrm{a}}$, Paul Jauch ${ }^{\mathrm{a}}$, Rudy Castro (retired) ${ }^{\mathrm{a}}$, Mark Ridley $^{\mathrm{a}}$, Brett Ungerman ${ }^{\mathrm{a}}$, Ralph Blenn ${ }^{\mathrm{a}}$, Evelyn Goodrich ${ }^{\mathrm{a}}$, Derek Dunlap ${ }^{\mathrm{a}}$, Duane Harp ${ }^{\mathrm{a}}$, Christopher Young ${ }^{\mathrm{a}}$, Tom Welsh ${ }^{\mathrm{a}}$ and NIRCam Team ${ }^{\mathrm{a}}$, and Tom Cinque ${ }^{\mathrm{d}}$ for help and support.

\section{REFERENCES}

1. S.D. Horner, M.J. Rieke, NIRCam team, "The Near Infrared Camera (NIRCam) for the James Webb Space Telescope (JWST)", Proc. SPIE 5487, 628 (2004).

2. J.E. Krist, K. Balasubramanian, C.A. Beichman, P.M. Echternach, K.M. Liewer, R.E. Muller, E. Seraby, S.B. Shaklan, J.T. Trauger, D.W. Wilson, S.D. Horner, Y. Mao, S.F. Somerstein, G. Vasudevan, D.M. Kelly, M.J. Rieke, "The JWST/NIRCam coronagraph: mask design and fabrication", Proc. SPIE 7440, p.74400W (2009)

3. J.E. Krist, "PROPER: an optical propagation library for IDL", Proc. SPIE 6675, p.66750P (2007)

4. T.B. Andersen, "Multiple-temperature lens design optimization," Proc. SPIE 2000, 2-6 (1993).

5. T.B. Andersen, "Some properties of Mertz type aspheric surfaces," Opt. Eng. 47, 093001 (2008).

6. D. Yang, M.E. Thomas, W.J. Tropf, S.G. Kaplan: "Infrared refractive index measurements using a new method", Proc. SPIE 4103, pp 42-52 (2000)

7. Y. Mao, D. Harrison, T. Richardson, B. Schultz, D. Taylor, L. W. Huff, S.D. Horner, D.M. Kelly, M.J. Rieke, "Cryogenic spectral performance of bandpass filters for NIRCam instruments", Proc. SPIE 7439, 74391D (2009)

8. CodeV ${ }^{\circledR}$ is a registered trademark of Optical Research Associates, Pasadena, California. 